\title{
Histone H3E73Q and H4E53A mutations cause recombinogenic DNA damage
}

\author{
Pedro Ortega ${ }^{1}$, Desiré García-Pichardo ${ }^{1}$, Marta San Martin-Alonso ${ }^{1}$, Ana G. Rondón ${ }^{1}$, Belén Gómez- \\ González ${ }^{1, *}$ and Andrés Aguilera ${ }^{1, *}$ \\ ${ }^{1}$ Centro Andaluz de Biología Molecular y Medicina Regenerativa (CABIMER), Universidad de Sevilla-CSIC-Universidad Pablo de \\ Olavide, Seville, Spain. \\ * Corresponding Authors: \\ Andrés Aguilera, Centro Andaluz de Biología Molecular y Medicina Regenerativa (CABIMER), Universidad de Sevilla-CSIC-Universidad \\ Pablo de Olavide, Seville, Spain; E-mail: aguilo@us.es; \\ Belén Gómez-González, Centro Andaluz de Biología Molecular y Medicina Regenerativa (CABIMER), Universidad de Sevilla-CSIC- \\ Universidad Pablo de Olavide, Seville, Spain; E-mail: gomezb@us.es
}

\begin{abstract}
The stability and function of eukaryotic genomes is closely linked to histones and to chromatin structure. The state of the chromatin not only affects the probability of DNA to undergo damage but also DNA repair. DNA damage can result in genetic alterations and subsequent development of cancer and other genetic diseases. Here, we identified two mutations in conserved residues of histone $\mathrm{H} 3$ and histone $\mathrm{H} 4$ (H3E73Q and H4E53A) that increase recombinogenic DNA damage. Our results suggest that the accumulation of DNA damage in these histone mutants is largely independent on transcription and might arise as a consequence of problems occurring during DNA replication. This study uncovers the relevance of H3E73 and H4E53 residues in the protection of genome integrity.
\end{abstract}

doi: $10.15698 /$ mic2020.07.723

Received originally: 06.03.2020;

in revised form: 14.04.2020,

Accepted 21.04.2020,

Published 24.04.2020.

Keywords: chromatin, DNA

replication, recombination, histone mutants.
Abbreviations:
AID - Activation-Induced Cytidine
Deaminase,
DSB - double-strand break,
$\mathrm{HU}$ - hydroxyurea,
MMS - methyl methane-sulfonate,
UV-ultra violet.

\section{INTRODUCTION}

Genetic instability is prevented through multiple processes to avoid cell death and tumorigenesis. Mechanisms ensuring replication fidelity as well as DNA damage checkpoints and repair pathways have evolved as a way to preserve genome integrity [1]. Accumulated evidence supports that most genetic instability sources arise from unrepaired DNA damage, such as double strand breaks (DSBs), or failures during DNA replication that can also ultimately lead to breaks. In this context, transcription is an important cause of replication stress, by directly or indirectly triggering transcription-replication conflicts [2].

The complex DNA organization into chromatin via packaging with histone and non-histone proteins influences all of these processes occurring at the DNA [3]. Chromatin structure exerts a major spatiotemporal control of DNA replication, repair and transcription processes thus affecting both the generation of endogenous damage as well as its efficient repair. Hence, whereas different chro- matin states can favor or impede DNA damage occurrence by enhancing or diminishing the accessibility of genotoxic agents, chromatin relaxation upon DNA damage promotes access of the repair machinery to the DNA lesion [4]. Moreover, chromatin can play an active role in regulating DNA repair, as first exemplified by the phosphorylation of the serine 189 of mammalian $\mathrm{H} 2 \mathrm{AX}$ histone variant (serine 129 phosphorylation of $\mathrm{H} 2 \mathrm{~A}$ in yeast, $\mathrm{P}-\mathrm{H} 2 \mathrm{~A}$ ), one of the earliest signals of the DNA damage checkpoint that expands up to $2 \mathrm{Mb}$ around DSBs initiating a cascade of recruitment of repair factors [5]. Since this modification was discovered, several other histone posttranslational modifications have been described to affect DNA damage repair, including the methylation of lysine and arginine, phosphorylation of serine and threonine and acetylation, ubiquitylation or sumoylation of lysine [6].

To explore the possible role of histones $\mathrm{H} 3$ and $\mathrm{H} 4$ residues in the maintenance of genome integrity in a systematic manner, we took advantage of a hyper-recombination 
A

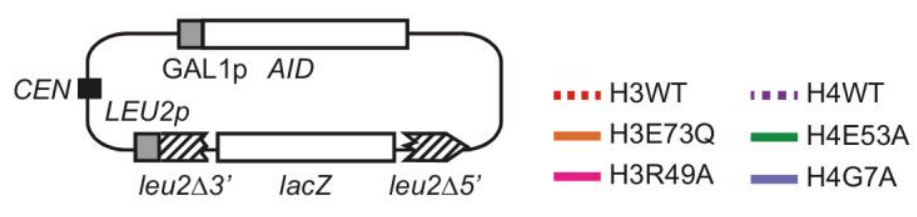

Recombinants (SC lacking leucine and tryptophan)

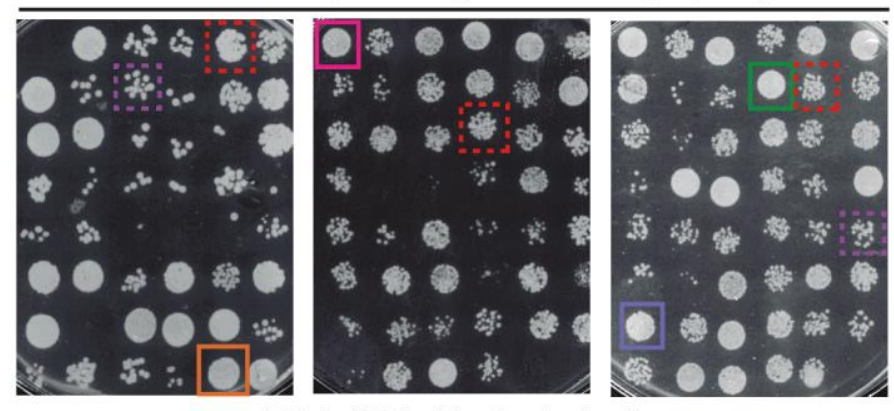

Totals (SC lacking tryptophan)

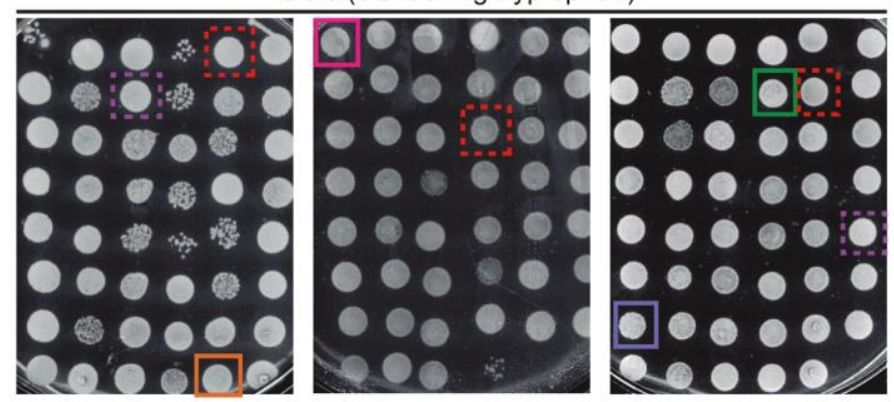

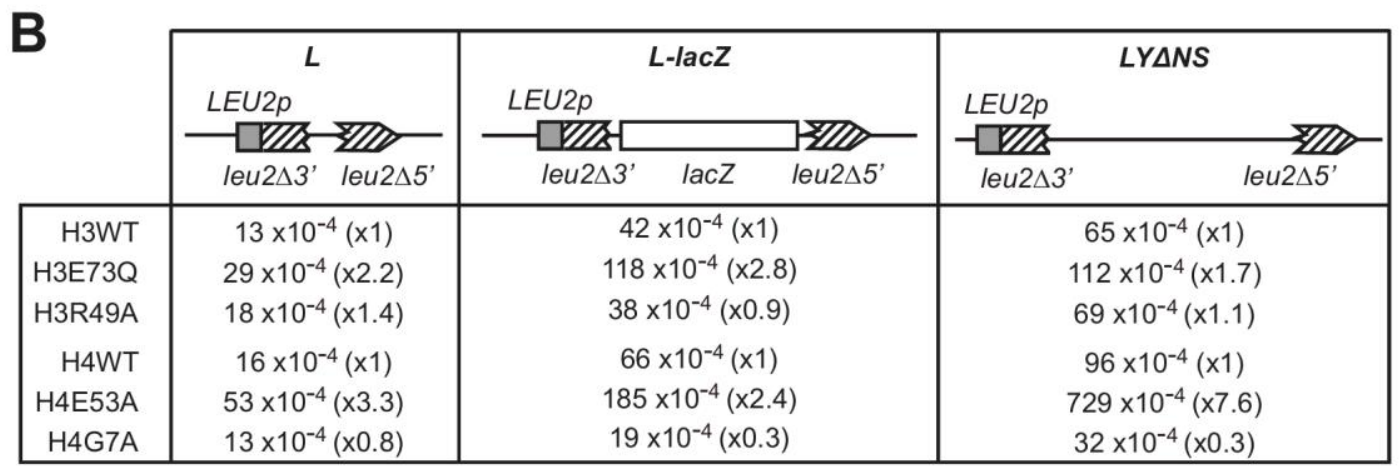

C

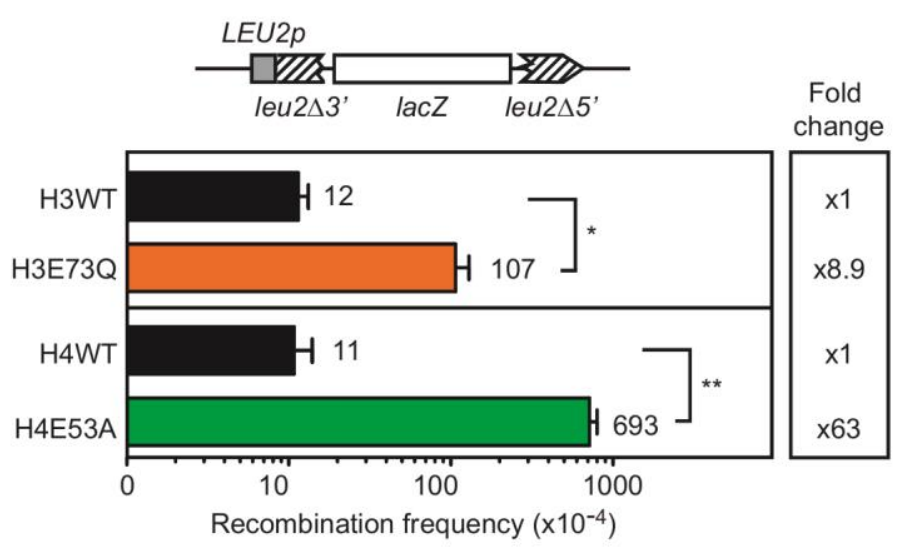


FIGURE 1: Histone H3E73Q and H4E53A mutants cause a hyper-recombination phenotype. (A) A scheme of the pLZGAID plasmid is shown. Visual analysis of direct-repeat recombination frequencies after AID overexpression in WT and histone mutant strains from the collection [8] transformed with pLZGAID. Similar dilutions of cultures grown in galactose media in 96-well-plates were plated in SC lacking leucine and tryptophan to detect Leu+ colonies (Recombinants) and in SC lacking tryptophan to visualize the total cells (Totals) and incubated for 3 days. Wild-type (H3WT), H3E73Q (H3E73Q), H3R49A (H3R49A), H4 wild-type (H4WT), H4E53A (H4E53A) and H4G7A (H4G7A) strains are pointed out. (B) A scheme of the L, L-lacZ, and LY $\triangle N$ S direct-repeat recombination system is shown. Analysis of median direct-repeat recombination frequencies in random colonies from H3 wild-type (H3WT), H3E73Q (H3E73Q), H3R49A (H3R49A), H4 wild-type (H4WT), H4E53A (H4E53A) and H4G7A (H4G7A) strains transformed with pRS316-L, pSCH204, and pRS316-LY $\triangle N$ S respectively. (C) A scheme of the L-lacZ direct-repeat recombination system is shown. Analysis of direct-repeat recombination frequencies in $\mathrm{H} 3$ wild-type (H3WT), H3E73Q (H3E73Q), H4 wildtype (H4WT) and H4E53A (H4E53A) strains transformed with pSCH204 ( $n=3)$. Means and SEM are plotted. ${ }^{*} p \leq 0.05, * * p \leq 0.01$ (two-tailed Student's t-test).

screening performed in a library of non-essential histone $\mathrm{H} 3$ and $\mathrm{H} 4$ mutants of Saccharomyces cerevisiae [7]. Here we describe two mutations in histones $\mathrm{H} 3$ and $\mathrm{H} 4$ ( $\mathrm{H} 3 \mathrm{E} 73 \mathrm{Q}$ and $\mathrm{H} 4 \mathrm{E} 53 \mathrm{~A}$ ) that increase the levels of spontaneous recombinogenic DNA damage. Our results suggest that damage accumulates as a consequence of problems during DNA replication, supporting a role of these histone residues in the maintenance of genome integrity by ensuring proper replication.

\section{RESULTS}

H3E73Q and H4E53A mutations increase the spontaneous levels of direct-repeat recombination

To study the relevance of particular histone residues in genetic stability we took advantage of a previously performed screening that analyzed the recombination frequency using a direct-repeat recombination system in a collection of non-essential histone $\mathrm{H} 3$ and $\mathrm{H} 4$ mutants in $S$. cerevisiae, in which one of the loci encoding for histone $\mathrm{H} 3$ and $\mathrm{H} 4$ genes (hht1-hhf1) was deleted, and the other one (hht2-hhf2) was replaced by a mutant copy [7]. This library contains 423 alleles that included each of the H3 and $\mathrm{H} 4$ residues substituted by alanine, original alanines substituted by serine as well as different substitutions of all modifiable residues by amino acids mimicking modified and unmodified states and sets of systematic deletions of the histone $\mathrm{N}$-terminal tails [8]. The screening was originally performed to identify histone residues that protect cells from accumulating DNA:RNA hybrids by selecting the mutations that enhanced recombination between direct repeats after the overexpression of AID (Activation-Induced Cytidine Deaminase) [7], which preferentially acts on the single-stranded (ss)DNA displaced by DNA:RNA hybrids [9]. Thus, histone mutations were selected only if they increased the appearance of recombinants after inducing AID overexpression, as assayed with the pLZGAID plasmid (Fig. 1A, [7]) that contains both the L-lacZ direct-repeat recombination system, consisting of two truncated direct repeats of the LEU2 gene with the bacterial lacZ gene placed in-between [10], and the AID cDNA under the control of the GAL promoter [9]. In galactose media, recombinational repair of AID-induced DNA breaks occurring between the repeats by Single-Strand Annealing (SSA) led to deletion of the lac $Z$ sequence and formation of a wild-type LEU2 allele, detectable as Leu+ recombinant colonies (Fig. 1A).
However, further experiments showed that some of the mutations enhanced the appearance of recombinant colonies not only after AID overexpression (galactose media) as the selection criteria (Fig. 1A), but also under conditions in which AID was not overexpressed (glucose media). These mutations were substitutions of the histone $\mathrm{H} 3$ glutamate 73 to glutamine (H3E73Q) or arginine 49 to alanine (H3R49A) and substitutions of the histone histone $\mathrm{H} 4$ glutamate 53 to alanine (H4E53A) or glycine 7 to alanine (H4G7A).

In a second phase of the screening, we studied the median frequency of recombination of random colonies from independent transformants with the L, L-lacZ and LY $\Delta N S$ direct-repeat recombination plasmid systems, which differ in the intervening sequence ( $30 \mathrm{bp}, 3 \mathrm{~Kb}$ and $5.6 \mathrm{~Kb}$ long, respectively) $[10,11]$. As shown in Fig. 1B, only H3E73Q and H4E53A mutants led to a significant increase in the recombination frequencies in all recombination systems and therefore, we proceeded with these two candidates. The increase was further confirmed with the L-lacZ system in both mutants. As shown in Fig. 1C, H3E73Q and H4E53A mutants led to a significant 8.9- and 63-fold increase in recombination frequencies with respect to the isogenic $H 3$ and $\mathrm{H} 4$ wild-type strains, respectively. Thus, $\mathrm{H} 3 \mathrm{E} 73 \mathrm{Q}$ and H4E53A mutations increase the levels of direct-repeat recombination regardless of AID, suggesting higher levels of spontaneous DNA breaks that are not associated with DNA:RNA hybrids.

\section{H3E73Q and H4E53A mutations increase spontaneous recombinogenic DNA damage}

To measure spontaneous DNA damage, we determined the levels of Rad52-YFP foci, indicative of repair centers that appear in the $S$ and $G 2$ phases of the cell cycle concurring with the coordination between recombination and replication [12]. As shown in Fig. 2A, both H4E53A and H3E73O mutants significantly enhanced the number of cells with Rad52-YFP foci (2.3- and 2.7-fold respectively) thus indicating that $\mathrm{H} 3 \mathrm{E} 73 \mathrm{Q}$ and $\mathrm{H} 4 \mathrm{E} 53 \mathrm{~A}$ mutations induce recombinogenic damage. We also analyzed the levels of $\mathrm{P}-\mathrm{H} 2 \mathrm{~A}$ as a marker of DSBs [5] (Fig. 2B). As a control, we also tested $\mathrm{P}-\mathrm{H} 2 \mathrm{~A}$ in the parental wild-type strain (BY4741) and after treatment with $0.05 \%$ methyl methane-sulfonate (MMS) during 1.5 hours, which led to a 2-fold increase. Whereas $\mathrm{H} 3 \mathrm{E} 73 \mathrm{Q}$ also led to a significant increase of P-H2A, H4E53A showed no differences with the wild-type. Thus, although both H3E73Q and H4E53A mutants accumulate 
A

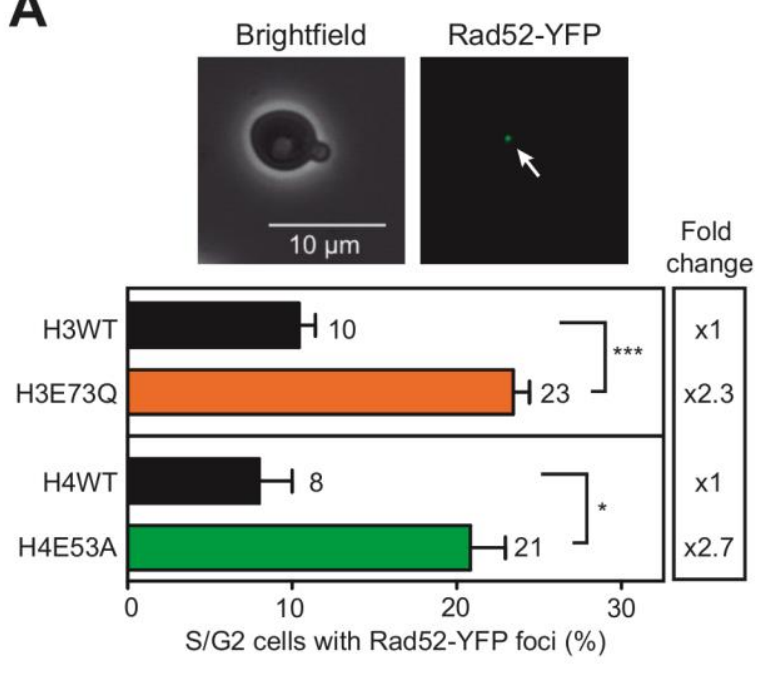

B

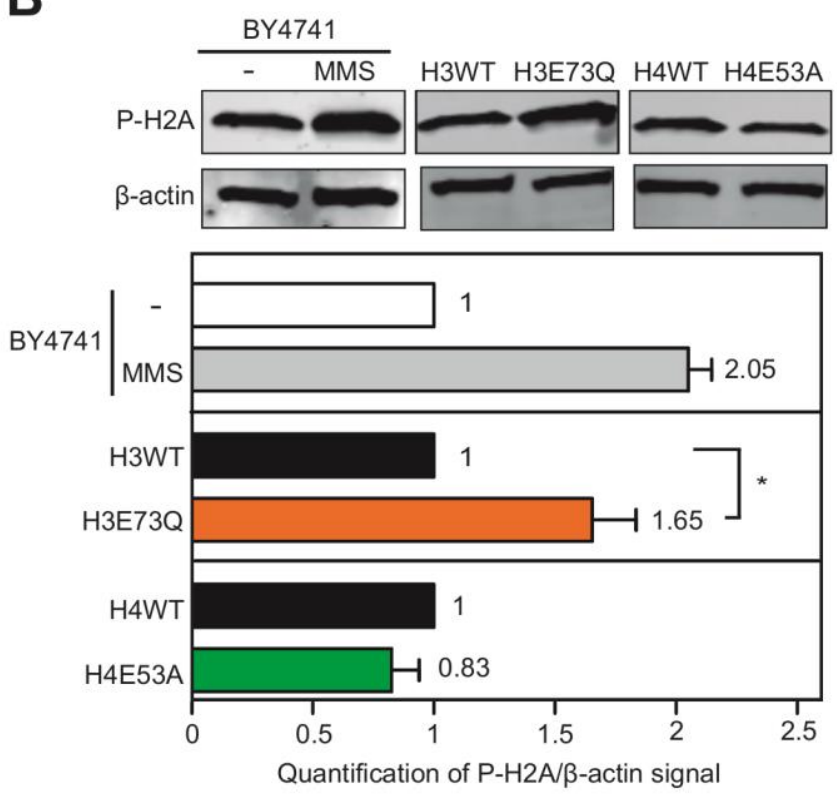

FIGURE 2: Histone H3E73Q or H4E53A mutants increase DNA damage. (A) Analysis of the percentage of S/G2 cells containing Rad52-YFP foci in H3 wild-type (H3WT), H3E73Q (H3E73Q), H4 wild-type (H4WT) and H4E53A (H4E53A) strains transformed with pWJ1344. A representative image of a cell with a Rad52-YFP focus is shown $(n=3)$. (B) Accumulation and quantification of the immunofluorescence signal of $P$ $\mathrm{H} 2 \mathrm{~A}$ in H3 wild-type (H3WT), H3E73Q (H3E73Q), H4 wild-type (H4WT) and H4E53A (H4E53A) strains detected by fluorescence-based western blot $(n=3)$. A representative fluorescence-based western blot and the BY4741 strain treated with $0.05 \%$ MMS for 1.5 hours as a control $(n=$ 2 ) is shown. Actin is shown as the loading control. Means and SEM are plotted in all panels. ${ }^{*} p \leq 0.05, * * * p \leq 0.001$ (two-tailed Student's t-test).

recombinogenic DNA damage, only $\mathrm{H} 3 \mathrm{E} 73 \mathrm{Q}$ led to a detectable increase in phosphorylated $\mathrm{H} 2 \mathrm{~A}$.

The hyper-recombination of H4E53A and H3E73Q mutants does not depend on transcription

Given that transcription is a major source of spontaneous DNA damage in the cell, we wondered whether the increase of recombinogenic DNA damage observed in H3E73Q and H4E53A mutants was associated with transcription. We used three direct-repeat recombination systems based on the previously described L-lacZ system, where the LEU2 promoter (LEU2p) was replaced by either the GAL1 inducible promoter (GAL1p) or the cell cyclespecific promoters (Fig. 3A) [13], HHF2p or CLB2p, which specifically activate transcription at the $S$ or $G 2$ phases, respectively [14, 15]. As shown in Fig. 3B and 3C, increased levels of recombination were obtained in H3E73Q and H4E53A mutants even when transcription was switched off (GAL1p in glucose) indicating that transcription has no major role in the hyper-recombination observed. H3E73Q hyper-recombination was further enhanced when transcription was switched on (galactose) (Fig. 3B), suggesting that transcription might explain part of the hyperrecombination phenotype of this mutant. Interestingly, recombination was only enhanced by transcription in the $S$ phase, but not in the $\mathrm{G} 2$ phase (Fig. 3B), suggesting that transcription-replication conflicts may contribute to the damage observed in this mutant. Instead, recombination was not stimulated by transcription in the H4E53A mutant, since the fold increase in recombination with respect to the wild-type was similar in glucose and in galactose (Fig. 3C). Moreover, a significant difference in the levels of recombinants was observed between the wild-type and the H4E53A mutant independent of the moment of the cell cycle in which the recombination system was transcribed (Fig. 3C) arguing against transcription as a source of DNA damage in this mutant. Importantly, the fact that both H3E73Q and H4E53A mutants led to increased levels of recombination when transcription was switched off indicates that the main source of recombinogenic DNA damage in these mutants does not depend on transcription.

\section{H3E73Q and H4E53A mutants increase DNA damage dur- ing replication}

Given that the $\mathrm{S}$ phase is when the DNA is most vulnerable during the cell cycle, we next wondered if the hyperrecombination phenotype observed could be a consequence of DNA damage originated during DNA replication. Thus, we analyzed by FACS the distribution of cells during the cell cycle. We noticed that cells of both $H 3 E 73 Q$ and H4E54A mutants accumulate in the S/G2 phase, suggesting DNA replication problems (Fig. 4A). We then decided to study cell cycle progression after synchronization in G1 with $\alpha$-factor. H4E53A cells presented a delay in cell cycle 
A

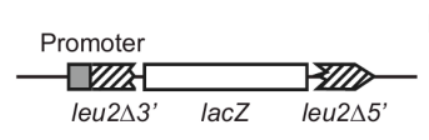
Promoter:
GAL1p (galactose-inducible)
HHF2p (S phase specific)
CLB2p (G2 phase specific)

B
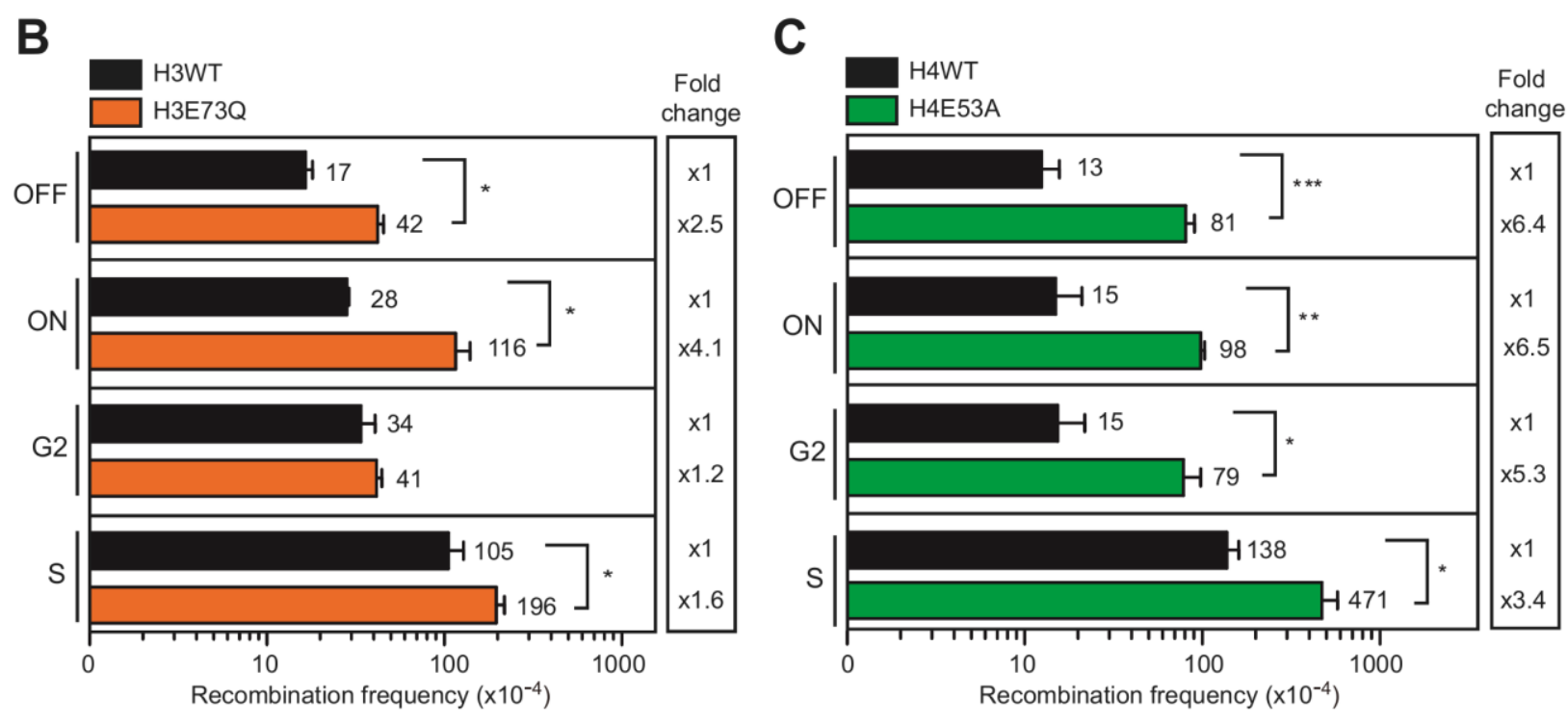

FIGURE 3: Hyper-recombination phenotype of H3E73Q and H4E53A mutants is not associated with defective transcription. (A) A scheme of the direct-repeat recombination system with different promoters is shown. GAL1 promoter (GAL1p) is induced in 2\% galactose (ON) and repressed in 2\% glucose (OFF). Transcription from CLB2 promoter (CLB2p) is restricted to G2 phase. Transcription from HHF2 promoter (HHF2p) is restricted to S phase. (B) Analysis of direct-repeat recombination frequencies in H3 wild-type (H3WT) and H3E73Q (H3E73Q) strains transformed with pARSLlacZGLB-in, pARSLlacZHLB-IN or pARSLlacZBLB plasmids with the direct-repeat recombination system under GAL1, HHF2, CLB2 promoter respectively ( $n=3)$. (C) Analysis of direct-repeat recombination frequencies in H4 wild-type (H4WT) and H4E53A (H4E53A) strains transformed with pARSLlacZGLB-in, pARSLlacZHLB-IN or pARSLlacZBLB plasmids with the direct-repeat recombination system under GAL1, HHF2, CLB2 promoters, respectively $(\mathrm{n}=3)$. Means and SEM are plotted in $(B)$ and $(C)$. ${ }^{*} p \leq 0.05, * * p \leq 0.01, * * * p \leq 0.001$ (two-tailed Student's t-test).

progression though the $\mathrm{S}$ phase clearly detectable between 60 and 100 minutes after G1 release (Fig. 4B). However, H3E73Q mutants did not respond to $\alpha$-factor and could not be included in the analysis (Fig. 4B).

If $\mathrm{H} 3 \mathrm{E} 73 \mathrm{Q}$ and $\mathrm{H} 4 \mathrm{E} 54 \mathrm{~A}$ mutations were affecting replication, we reasoned that challenging these mutants with genotoxic agents that generate damage during the S/G2 phase would affect their growth. To test that idea, we analyzed growth in media containing either hydroxyurea (HU), which depletes the dNTP pools, or MMS, a DNA alkylating agent. In addition, we tested sensitivity to ultraviolet light (UV), which causes DNA damage throughout the cell cycle. In the single histone mutants, we did not detect large effects in cell viability with any of the treatments suggesting that the amount of DNA damage generated was low and efficiently counteracted by the repair systems (Fig. 4C). Thus, given that the checkpoint machinery is required for cell survival upon DNA damage, we decided to further challenge these histone mutants and study the genetic interaction with mutations in the DNA damage checkpoint, a strategy previously used to reveal the role of the checkpoint in transcription-associated DNA damage [16]. For this, we generated double mutants of $\mathrm{H} 3 \mathrm{E} 73 \mathrm{Q}$ and H4E54A with the rad24 $\triangle$ checkpoint mutant and analyzed the growth after HU, MMS or UV exposure. As shown in Fig. 4C, rad24 $\triangle$ impaired survival of H4E53A or H3E73Q to all the genotoxic agent treatments. This argues that both H4E53A and H3E73Q mutants accumulate lesions that require the DNA damage checkpoint for survival under further stress. Altogether, these results suggest that hyper-recombination of H3E73Q and H4E53A mutants is the result of DNA damage accumulation likely during replication.

\section{DISCUSSION}

In this study, we have identified and characterized two mutations of histone $\mathrm{H} 3$ and $\mathrm{H} 4$ (H3E73Q and H4E53A) that increase genome instability. $\mathrm{H} 3 \mathrm{E} 73 \mathrm{Q}$ and $\mathrm{H} 4 \mathrm{E} 53 \mathrm{~A}$ residues are conserved and located on the disk surface of the nucleosome thus not interacting with DNA (Fig. 5). Both mutations caused hyper-recombination (higher in H4E53A) and increased spontaneous DNA damage. Although evidence from our and other labs in the last two decades has shown that transcription is a major cause of genome instability [17], we show that hyper-recombination is independent of in H3E73Q and H4E53A (Fig. 3). Both, H3E73Q and H4E53A mutations increased the percentage of cells with 
A
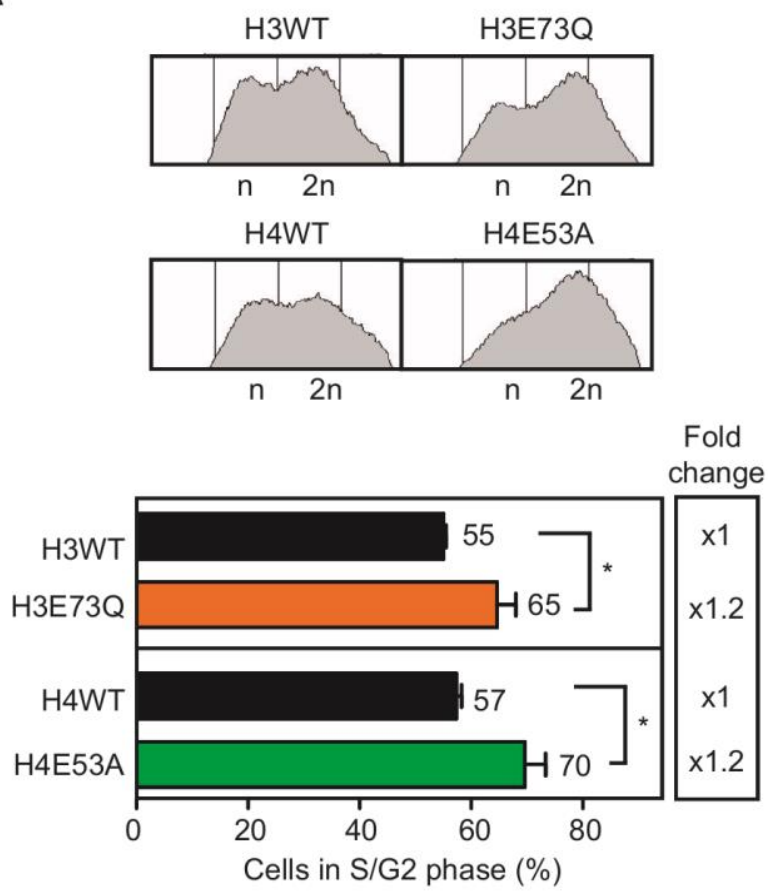

B
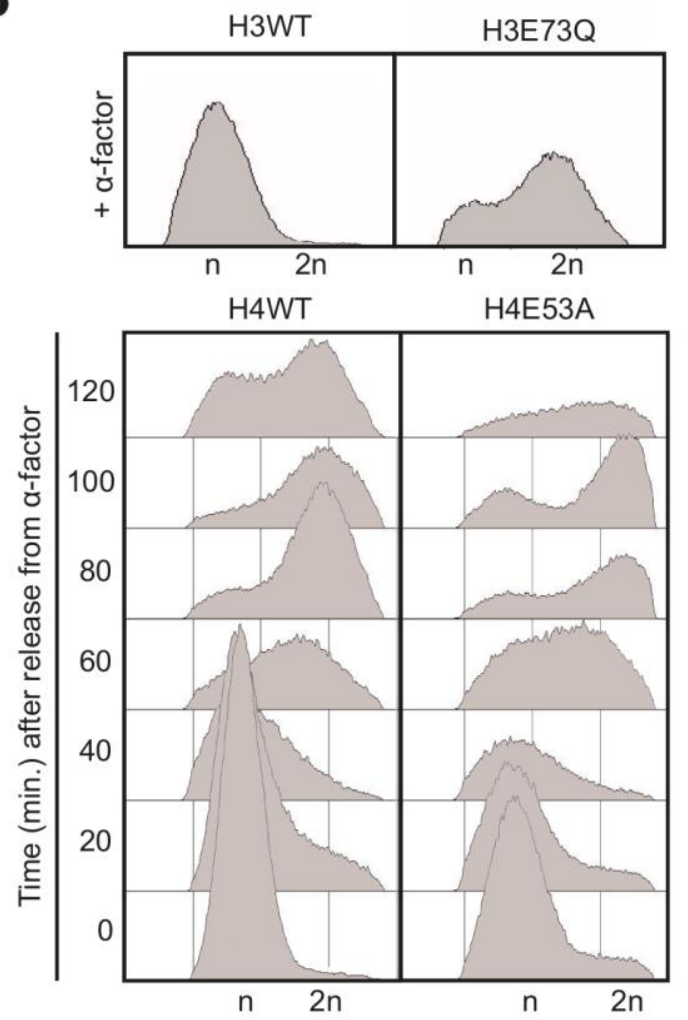

C

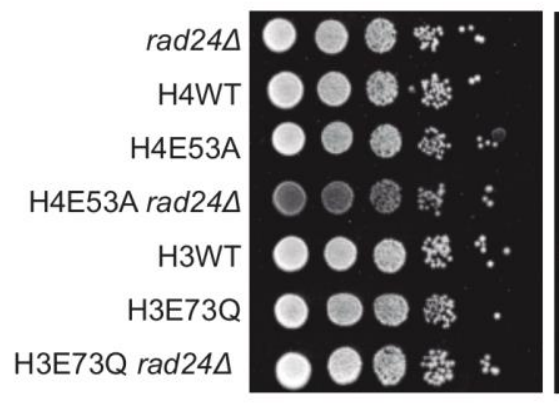

$\mathrm{HU}(150 \mathrm{mM})$

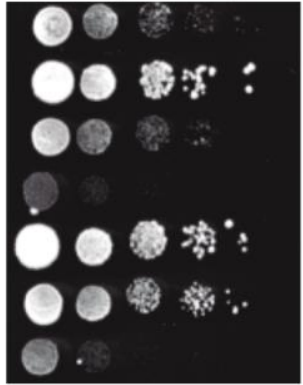

MMS $(0.02 \%)$

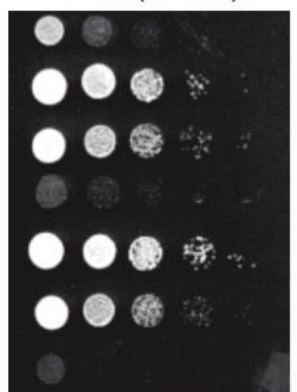

UV $\left(40 \mathrm{~J} / \mathrm{m}^{2}\right)$

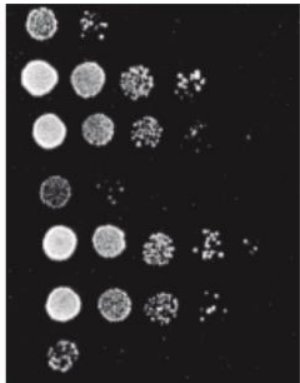

FIGURE 4: Histone H3E73Q and H4E53A mutants increase replication stress. (A) Analysis and quantification of cell cycle phases in asynchronous cultures of H3 wild-type (H3WT), H3E73Q (H3E73Q), H4 wild-type (H4WT) and H4E53A (H4E53A) strains by FACS ( $n=3$ ). (B) Analysis of cell cycle progression in H3 wild-type (H3WT), H3E73Q (H3E73Q), H4 wild-type (H4WT) and H4E53A (H4E53A) strains by FACS. (C) Sensitivity

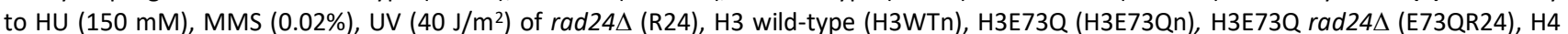
wild-type (H4WTn), H4E53A (H4E53An) and H3E73Q rad24A (E53AR24) strains coming from a crossing H3E73Q and H4E53A with W303-1B rad24 $\Delta$. Similar results were obtained with different spores from the same genetic cross. Means and SEM are plotted in (A). ${ }^{*} \mathrm{p} \leq 0.05$. (twotailed Student's t-test).

Rad52 foci, cells in the S/G2 phases, and sensitivity to genotoxic agents when the DNA damage checkpoint was inactivated by deleting RAD24. Altogether, the data suggests that these two histone residues have a role in preventing recombinogenic DNA damage during the $S$ phase.

Interestingly, $\mathrm{H} 3 \mathrm{E} 73 \mathrm{Q}$ and $\mathrm{H} 4 \mathrm{E} 53 \mathrm{~A}$ also conferred specific phenotypes. Thus, only $\mathrm{H} 3 \mathrm{E} 73 \mathrm{Q}$, but not H4E53A led to increased P-H2A levels, likely reflecting the accumulation of spontaneous DSBs. There was not a hyperrecombination phenotype in $\mathrm{H} 3 \mathrm{E} 73 \mathrm{Q}$ when the promoter was active in $\mathrm{G} 2$ but since a 2.5 -fold recombination increase was still observed when switching off transcription (GAL1p in glucose), it seems that transcription is not the major driver of DNA damage in neither of the mutants. The fact that $\mathrm{H} 3 \mathrm{E} 73 \mathrm{Q}$ cells could not be synchronized with $\alpha$-factor might be due to loss of heterochromatin silencing since the H3E73 residue lies in the LRS (Loss of rDNA Silencing) domain. This domain is necessary for sirtuins to deacetylate heterochromatin keeping it silenced [18-20] and when the $H M L$ and $H M R$ heterochromatic regions are 
H3E73

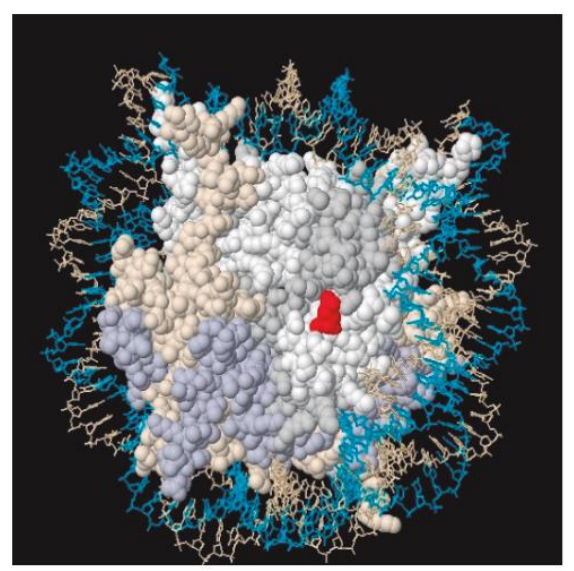

H4E53

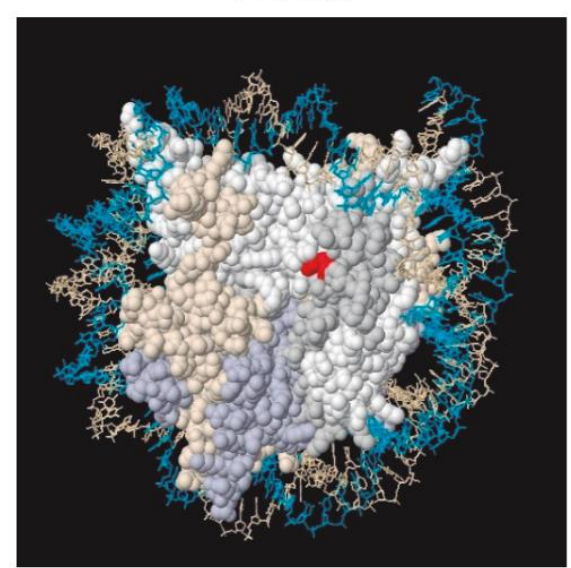

FIGURE 5: Location of H3E73Q and H4E53A mutations. 3D view of the nucleosome crystal structure of the yeast nucleosomes with H3E73 and H4E53 residues colored in red. The images were obtained from www.histonehits.org [18]. expressed, haploid yeasts are not able to respond to $\alpha$-factor [21]. Indeed, H3K73Q has been reported to affect $H M R$ and telomeric silencing [18]. However, this effect is not complete and indeed, we were able to cross this mutant to generate double mutants with rad24D (Fig. 4C). Furthermore, it is also possible that this partial suppression of silencing is involved in the hyper-recombination conferred by $\mathrm{H} 3 \mathrm{E} 73 \mathrm{Q}$ since heterochromatin de-silencing can alter replication timing, that has been previously associated with genome instability $[22,23]$. Similarly, the increase of DSBs (Fig. 2B), S/G2 cells containing Rad52 foci (Fig. 2A) and cells in the S/G2 phase observed in H3E73Q mutant (Fig. 4A) could also be due to the reported defective silencing of telomeric regions [18]. These observations will explain the DNA damage sensitivity observed when we deleted RAD24 in H3E73Q mutant. However, provided the potential pleotropic effect of histone modifications in silencing, gene expression and DNA transactions, further detailed analysis would be necessary to define the specific molecular mechanisms by which the H3E73Q mutation compromises genome integrity.

By contrast, the H4E53A mutant responds to $\alpha$-factor and shows a delay in $\mathrm{S}$ phase progression (Fig. 4B), which supports that DNA damage accumulates during replication. H4E53A might be directly or indirectly affecting the replication process itself, in this case clearly in a transcriptionindependent manner (Fig. 3C). Interestingly, the H4E53 residue has been reported to interact with Cac1, the largest subunit of the Chromatin Assembly Factor 1 (CAF-1), which, together with Asf1, promotes histone $\mathrm{H} 3$ and $\mathrm{H} 4$ deposition onto newly synthesized DNA during replication, what is essential for proper S-phase progression [24, 25]. Thus, it is tempting to speculate that H4E53A might impair the interaction with Cac1 affecting S-phase progression and leading to the observed DNA damage accumulation. However, again further detailed analysis would be necessary to define the specific molecular mechanisms by which this mutation compromises genome integrity.

In summary, our study uncovered a key function of the conserved H3E73 and H4E53 histone residues in the maintenance of genome integrity by preventing the for- mation of recombinogenic DNA damage, adding new light into our understanding of the role of histones in the mechanisms of genome integrity that would need to be explored further.

\section{MATERIALS AND METHODS \\ Yeast strains and media}

BY4741, H3WT, H4WT, H3E73Q, H3R49A, H4E53A and H4G7A yeast strains have been reported previously $[7,8]$. H3E73Q and H4E53A were crossed with W303-1B rad24D (WR24-6C) [16] to obtain hht1-hhf1::NatMX4 hht2-hhf2::[H3]-URA3 (H3WTn), hht1-hhf1::NatMX4 hht2-hhf2::[H4]-URA3 (H4WTn), TRP1 hht1-hhf1::natMX4 hht2-hhf2::[E73Q]-URA3 (H3E73Qn) and hht1-hhf1:: natMX4 hht2-hhf2::[E53A]-URA3 (H4E53An), rad24A::TRP1 hht1-hhf1:: natMX4 (R24), rad24A::TRP1 hht1hhf1::natMX4 hht2-hhf2::[E73Q]-URA3 (E73QR24) and rad24L::TRP1 hht1-hhf1:: natMX4 hht2-hhf2::[E53A]-URA3 (E53AR24).

Media used in this study: YPAD (1\% yeast extract, $2 \%$ bacto-peptone, $2 \%$ glucose, $20 \mathrm{mg} / \mathrm{L}$ adenine), SD $(0.17 \%$ yeast nitrogen base (YNB) without amino acids nor ammonium sulfate, $0.5 \%$ ammonium sulfate and supplemented with amino acids. The absence of amino acid/s is specified when required), SC (SD containing $2 \%$ glucose) and SPO ( $1 \%$ potassium acetate, $0.1 \%$ yeast extract, $0.005 \%$ glucose). Solid media were prepared adding $2 \%$ agar before autoclaving.

Yeast strains were freshly defrosted from stocks and grown at $30^{\circ} \mathrm{C}$. All experiments were performed at $30^{\circ} \mathrm{C}$.

\section{Plasmids}

All plasmids used in this study were previously reported. pWJ1344 contains the Rad52::YFP construct [12]. pRS316-L is a centromeric plasmid containing a leu $2 \Delta 3^{\prime}::$ leu $2 \Delta 5^{\prime}$ directrepeat construct [11]. pRS314L/acZ is a centromeric plasmid containing the leu $2 \Delta 3^{\prime}::$ leu $2 \Delta 5^{\prime}$ direct-repeat construct with the lacZ gene in between the repeats [10]. pRS316-LY $\triangle N S$ is a centromeric plasmid containing the leu2 $\Delta 3^{\prime}:$ :leu $2 \Delta 5^{\prime}$ directrepeat construct with a fragment of the Ylp5 plasmid in between the repeats [11]. pLZGAID is a centromeric plasmid containing containing the $L$ lacZ construct and the AID under the GAL1 promoter [7]. pARSLlacZGLB-IN, pARSLlacZHLB-IN and pARSLlacZBLB-IN are centromeric plasmids containing the 
LlacZ construct transcribed from the GAL1, HHF2 and CLB2 promoters, respectively $[13,26]$.

\section{Genetic analysis of recombination}

For the recombination assays with the direct-repeat systems, cells were grown on plates with SC lacking tryptophan for 3 to 4 days. Recombinants were selected on plates with SC medium lacking leucine. In Fig. 1A, qualitative recombination frequencies of different mutants from the collection of nonessential histone $\mathrm{H} 3$ and $\mathrm{H} 4$ mutants were obtained. In Fig. 1B recombination frequencies were the median value of a total of at least eleven colonies coming from three independent transformants. In Fig. $1 \mathrm{C}$ and 3, recombination frequencies were obtained by fluctuation tests as the median value of six independent colonies isolated from plates with SC medium. The final frequency given for each strain and condition is the mean and SEM deviation of three to four median values, as described previously [27].

\section{Analyses of Rad52-YFP foci}

Rad52-YFP foci were counted in more than 200 S/G2 cells transformed with pWJ1344. Cells were visualized in Leica DC 350F. The mean and SEM of three different experiments were plotted.

\section{Western blot analysis}

$10 \mathrm{~mL}$ of each strain culture at 0.7 (O.D. $600 \mathrm{~nm}$ ) growing in SC were recovered and kept on ice. The culture was centrifuged and proteins were extracted from pellets by adding $200 \mu \mathrm{L}$ of cold $10 \%$ TCA and $200 \mu \mathrm{L}$ of glass beads by vortexing 7 times during 20 seconds each time at $4^{\circ} \mathrm{C}$. Supernatant was recovered and beads were washed twice with $200 \mu \mathrm{L}$ of cold $10 \%$ TCA. Samples were centrifuged 10 minutes at $3000 \mathrm{rpm}$ and supernatants were discarded. The remaining pellet was resuspended using $100 \mu \mathrm{L}$ of loading Buffer (62.5 mM Tris-HCl $\mathrm{pH}$ 6.8, 25\% glycerol, $2 \%$ SDS, $0.01 \%$ water-dilluted Bromophenol Blue, $5 \% \beta$-mercaptoethanol), $50 \mu \mathrm{L}$ of water and $50 \mu \mathrm{L}$ of $1 \mathrm{M}$ Tris (not-adjusted $\mathrm{pH}$ ). Prior to gel loading samples were boiled for 5 minutes and centrifuged 10 minutes at 3000 $\mathrm{rpm}$ at room temperature. Proteins were separated on BIORAD mini-Protean TGX Gel and wet-transferred to PVDF membranes (Immobilon-FL, Milipore). Antibody 8332 (Abcam, UK) for $\beta$-actin was used at a 1:1000 and antibody 39271 (BIOMOL) for P-H2A was used at 1:2000 dilution, both in 1x TBS$0,1 \%$-Tween and incubated 1 hour with fluorescence secondary antibody IRDW $600 \mathrm{CW}$ or $800 \mathrm{CW}$ for signal detection. The actin signal is shown as a loading control and its levels were used to normalize the amount of P-H2A for each transformant The mean and SEM of three different experiments were plotted.

\section{Cell cycle synchronization and flow cytometry}

Cells were arrested in the G1 phase with $2.5 \mu \mathrm{M}$ of $\alpha$-factor mating pheromone. Approximately $1 \mathrm{~mL}\left(10^{7}\right.$ cells) were col- lected from G1-arrested or asynchronous cultures at the indicated time points centrifuge 1 minute at $5000 \mathrm{rpm}$ and washed twice with sodium citrate $50 \mathrm{mM} \mathrm{pH}$ 7.5. Cells were resuspended in $1 \mathrm{~mL}$ of sodium citrate $50 \mathrm{mM} \mathrm{pH} 7.5$ and incubated one hour with $25 \mu \mathrm{L}$ of RNase $A(10 \mathrm{mg} / \mathrm{mL})$ and one hour with $20 \mathrm{mg} / \mathrm{mL}$ of proteinase $\mathrm{K}$ at $50^{\circ} \mathrm{C}$. Cells were incubated 1 hour at $50^{\circ} \mathrm{C}$ after the addition of $1 \mathrm{~mL}$ sodium citrate $50 \mathrm{mM} \mathrm{pH} 7.5$ containing $16 \mu \mathrm{g} / \mathrm{mL}$ propidium iodide. Cell cycle distribution was analysed using a FACSCalibur system (Becton-Dickinson).

\section{Genotoxic-sensitivity assays}

Mid-log cultures were grown in YPAD medium. 10-fold dilutions of the culture prepared in sterile water were plated on solid YPAD medium containing the drugs at the indicated concentrations. UV irradiation was performed with the dried plates. Plates were incubated during 3 days (in the dark for UV-irradiated plates).

\section{AUTHOR CONTRIBUATION}

P.O., D. G.-P and M. SM.-A. performed the experiments; P.O., A. R., D. G.-P., B.G.-G., and A.A. designed the experiments. P.O., B.G.-G., and A.A. wrote the manuscript. All authors read, discussed, and agreed with the final version of this manuscript.

\section{ACKNOWLEDGMENTS}

Research was supported by the European Research Council (ERC2014 AdG669898 TARLOOP), the Spanish Ministry of Economy and Competitiveness (BFU2016-75058-P), the Foundation Vencer el Cáncer and the European Union (FEDER). P. O. was supported by a predoctoral training grant from the Spanish Ministry of Economy and Competitiveness and B.G.-G. by the Spanish Association Against Cancer (AECC).

\section{CONFLICT OF INTEREST}

The authors declare no competing interests.

\section{COPYRIGHT}

(C) 2020 Ortega et al. This is an open-access article released under the terms of the Creative Commons Attribution (CC BY) license, which allows the unrestricted use, distribution, and reproduction in any medium, provided the original author and source are acknowledged.

Please cite this article as: Pedro Ortega, Desiré García-Pichardo, Marta San Martin-Alonso, Ana G. Rondón, Belén Gómez-González and Andrés Aguilera (2020). Histone H3E73Q and H4E53A mutations cause recombinogenic DNA damage. Microbial Cell 7(7): 190-198. doi: 10.15698/mic2020.07.723

safeguard against harmful DNA-RNA hybrids of different origins. EMBO Rep 20(9): e47250. doi: 10.15252/embr.201847250

3. Campos El, Reinberg D (2009). Histones: annotating chromatin. Annu Rev Genet 43: 559-599. doi 10.1146/annurev.genet.032608.103928

\section{REFERENCES}

1. Ciccia A, Elledge SJ (2010). The DNA damage response: making it safe to play with knives. Mol Cell 40(2): 179-204. doi: 10.1016/j.molcel.2010.09.019

2. Barroso S, Herrera-Moyano E, Munoz S, Garcia-Rubio M, GomezGonzalez B, Aguilera A (2019). The DNA damage response acts as a 
4. Stadler J, Richly H (2017). Regulation of DNA Repair Mechanisms: How the Chromatin Environment Regulates the DNA Damage Response. Int J Mol Sci 18(8): E1715. doi: 10.3390/ijms18081715

5. Rogakou EP, Pilch DR, Orr AH, Ivanova VS, Bonner WM (1998). DNA double-stranded breaks induce histone $\mathrm{H} 2 \mathrm{AX}$ phosphorylation on serine 139. J Biol Chem 273(10): 5858-5868. doi: 10.1074/jbc. 273.10 .5858

6. Suganuma T, Workman JL (2011). Signals and combinatorial functions of histone modifications. Annu Rev Biochem 80:473-499. doi: 10.1146/annurev-biochem-061809-175347

7. Garcia-Pichardo D, Canas JC, Garcia-Rubio ML, Gomez-Gonzalez B, Rondon AG, Aguilera A (2017). Histone Mutants Separate R Loop Formation from Genome Instability Induction. Mol Cell 66(5): 597-609 e595. doi: 10.1016/j.molcel.2017.05.014

8. Dai J, Hyland EM, Yuan DS, Huang H, Bader JS, Boeke JD (2008). Probing nucleosome function: a highly versatile library of synthetic histone $\mathrm{H} 3$ and $\mathrm{H} 4$ mutants. Cell 134(6): 1066-1078. doi: 10.1016/j.cell.2008.07.019

9. Gomez-Gonzalez B, Aguilera A (2007). Activation-induced cytidine deaminase action is strongly stimulated by mutations of the THO complex. Proc Natl Acad Sci USA 104(20): 8409-8414. doi: 10.1073/pnas.0702836104

10. Chavez S, Aguilera A (1997). The yeast HPR1 gene has a functional role in transcriptional elongation that uncovers a novel source of genome instability. Genes Dev 11(24): 3459-3470. doi: 10.1101/gad.11.24.3459

11. Prado F, Piruat JI, Aguilera A. (1997) Recombination between DNA repeats in yeast hpr1delta cells is linked to transcription elongation. EMBO J 16(10):2826-35. doi: 10.1093/emboj/16.10.2826

12. Lisby M, Rothstein R, Mortensen UH (2001). Rad52 forms DNA repair and recombination centers during $S$ phase. Proc Natl Acad Sci USA 98(15): 8276-8282. doi: 10.1073/pnas.121006298

13. Prado F, Aguilera A (2005). Impairment of replication fork progression mediates RNA polll transcription-associated recombination. EMBO J 24(6): 1267-1276. doi: 10.1038/sj.emboj. 7600602

14. Hereford LM, Osley MA, Ludwig TR, 2nd, McLaughlin CS (1981). Cell-cycle regulation of yeast histone mRNA. Cell 24(2): 367-375. doi: 10.1016/0092-8674(81)90326-3

15. Maher M, Cong F, Kindelberger D, Nasmyth K, Dalton S (1995). Cell cycle-regulated transcription of the CLB2 gene is dependent on Mcm1 and a ternary complex factor. Mol Cell Biol 15(6): 3129-3137. doi: 10.1128/mcb.15.6.3129

16. Gomez-Gonzalez B, Felipe-Abrio I, Aguilera A (2009). The S-phase checkpoint is required to respond to R-loops accumulated in $\mathrm{THO}$ mutants. Mol Cell Biol 29(19): 5203-5213. doi: 10.1128/MCB.0040209

17. Gomez-Gonzalez B, Aguilera A (2019). Transcription-mediated replication hindrance: a major driver of genome instability. Genes Dev 33(15-16): 1008-1026. doi: 10.1101/gad.324517.119

18. Huang H, Maertens AM, Hyland EM, Dai J, Norris A, Boeke JD, Bader JS (2009). HistoneHits: a database for histone mutations and their phenotypes. Genome research 19(4): 674-681. doi: 10.1101/gr.083402.108

19. Norris A, Bianchet MA, Boeke JD (2008). Compensatory interactions between Sir3p and the nucleosomal LRS surface imply their direct interaction. PLoS Genet 4(12): e1000301. doi: 10.1371/journal.pgen.1000301

20. Manning BJ, Peterson CL (2014). Direct interactions promote eviction of the Sir3 heterochromatin protein by the SWI/SNF chromatin remodeling enzyme. Proc Natl Acad Sci USA 111(50): 17827-17832. doi: 10.1073/pnas.1420096111

21. Merlini L, Dudin O, Martin SG (2013). Mate and fuse: how yeast cells do it. Open Biol 3(3): 130008. doi: 10.1098/rsob.130008

22. Yoshida K, Bacal J, Desmarais D, Padioleau I, Tsaponina O, Chabes A, Pantesco V, Dubois E, Parrinello H, Skrzypczak M, Ginalski K, Lengronne A, Pasero $P$ (2014). The histone deacetylases sir2 and rpd3 act on ribosomal DNA to control the replication program in budding yeast. Mol Cell 54(4): 691-697. doi: 10.1016/j.molcel.2014.04.032

23. Foss EJ, Lao U, Dalrymple E, Adrianse RL, Loe T, Bedalov A (2017). SIR2 suppresses replication gaps and genome instability by balancing replication between repetitive and unique sequences. Proc Natl Acad Sci USA 114(3): 552-557. doi: 10.1073/pnas.1614781114

24. Tyler JK, Collins KA, Prasad-Sinha J, Amiott E, Bulger M, Harte PJ, Kobayashi R, Kadonaga JT (2001). Interaction between the Drosophila CAF-1 and ASF1 chromatin assembly factors. Mol Cell Biol 21(19): 6574-6584. doi: 10.1128/mcb.21.19.6574-6584.2001

25. Liu WH, Roemer SC, Zhou Y, Shen ZJ, Dennehey BK, Balsbaugh JL, Liddle JC, Nemkov T, Ahn NG, Hansen KC, Tyler JK, Churchill ME (2016). The Cac1 subunit of histone chaperone CAF-1 organizes CAF-1$\mathrm{H} 3 / \mathrm{H} 4$ architecture and tetramerizes histones. Elife 5: e18023. doi: 10.7554/eLife.18023

26. Wellinger RE, Prado F, Aguilera A (2006). Replication fork progression is impaired by transcription in hyperrecombinant yeast cells lacking a functional THO complex. Mol Cell Biol 26(8): 3327-3334. doi: 10.1128/mcb.26.8.3327-3334.2006

27. Aguilera A, Klein HL (1989). Genetic and molecular analysis of recombination events in Saccharomyces cerevisiae occurring in the presence of the hyper-recombination mutation hpr1. Genetics 122(3): 503-517. PMID: 2668113 